\title{
Viscometric Studies of Nicotinic Acid in Binary Aqueous Mixtures of D-Lactose
}

\author{
RAVI SHARMA, SONIKA and R.C.THAKUR* \\ Department of Chemistry, School of Chemical Engineering and Physical Sciences, Lovely \\ Professional University, Phagwara, Punjab, India. \\ ${ }^{*}$ Corresponding author E-mail: drthakurchem @ gmail.com \\ http://dx.doi.org/10.13005/ojc/330352
}

(Received: February 22, 2017; Accepted: April 14, 2017)

\begin{abstract}
Relative viscosities of nicotinic acid in D-lactose + water have been measured at different temperatures $(298.15,303.15,308.15,313.15) \mathrm{K}$. The analysis of the viscosity data has been done by using Jones-Dole equation and A and B-coefficients of Jones-Dole equation have been interpreted in terms of solute-solute and solute-solvent interactions. The present study concludes the structure making nature of nicotinic acid in binary aqueous mixtures of D-lactose.
\end{abstract}

Keywords: Relative viscosity, B-coefficient and Structure maker

\section{INTRODUCTION}

The study of viscometric properties of various solutions is one of the most interesting areas in physical chemistry and this work has been expanded in various directions in last few years. The different structure and molecular properties of solutions can be determined using viscometric studies $^{1-3}$. Different kinds of interactions exist in the solutions and out of those solute - solute and solute - solvent interactions are the current areas of interest. These interactions explain the structure making and breaking nature of solute in the given solvent. Accurate aqueous data is needed over wide range of temperature, pressure and composition in many fields besides chemistry such as biological systems and pharmaceutical industries. The literature survey shows that a number of studies have been conducted on many biomolecules but a little attentions was given for the study of viscometric properties of nicotinic acid in aqueous and binary aqueous mixtures of D-lactose ${ }^{4-16}$. Nicotinic acid is also known as vitamin $B_{3}$ and is water soluble, colourless and also used in pharmacological doses. It has a great role in DNA repairing and production of steroid hormones. Also, it is used as an additive in food, forage and cosmetics. Lactose is one of the major parts of diet especially for infants and is also used to sweeten stout beer, called a milk stout or a cream stout. Lactose because of its physical 
properties i.e. compressibility, and low price is also added to pills as filler and also for dilution of heroin.

\section{Materials and methods}

Both the reagents nicotinic acid $\left(\mathrm{C}_{6} \mathrm{H}_{5} \mathrm{NO}_{2}\right.$, M.W.123.11g) and D-Lactose $\left(\mathrm{C}_{12} \mathrm{H}_{24} \mathrm{O}_{12}\right.$, M.W.320.32g) were of A.R grade and used after drying over calcium oxide for more than 24 hours. Both the reagents were procured from S.D Fine-Chem Limited. The triple distilled deionized water with a $\mathrm{pH}$ of 7.1 and conductivity ranging from $1-2 \mu \mathrm{s}-\mathrm{cm}^{-1}$ was used during the experiment for the preparation of the solutions and various other compositions. Molal solutions were prepared using an analytical balance (FA2204B, Shanghai Jingke, China) at 298.15 K with an uncertainty of $\pm 0.0001 \mathrm{~g}$ and concentrations were prepared with an uncertainty of 0.0001 mol.kg"1. The chemicals used in the present study were dried over calcium oxide for a period of more than $24 \mathrm{~h}$. and molal solutions $(0.01,0.03,0.05,0.07,0.1$ and 0.15$)$ mol.kg"1 of nicotinic acid in binary aqueous solutions of D-lactose $(0.05,0.15,0.25$ and 0.35$) \mathrm{mol} \mathrm{kg}^{-1}$ were prepared for determining densities and viscosities.

The molal concentration, $(m)$ was converted into molar concentration, (c) by using the standard expression:

$$
c=\frac{m \rho 1000}{1000+m M}
$$

Where $\rho$ is the density of sample and $M$ is the molecular weight of the nicotinic acid. The densities, $\rho$ for all the solutions were measured by using an Anton Paar DSA 5000 M density and sound velocity meter. The instrument was calibrated with triple distilled and degassed water in the experimental temperature range. The instrument was controlled to $\pm 1 \times 10^{-3} \mathrm{~K}$ by built-in Peltier device as density is extremely sensitive to temperature. The uncertainty of the density was found to be $\pm 3 \times 10^{-3}$ $\mathrm{kg} \cdot \mathrm{m}^{-3}$. The viscosities and the relative viscosities of all the solutions of nicotinic acid in water as well as in binary aqueous mixtures of D-lactose were determined by using an ubbelohde viscometer having a flow time of 57 seconds for pure water at 303.15 K. The procedure was repeated till three successive values were determined within a range of \pm 0.1 second. The effect of temperature was observed using water thermostat $( \pm 0.01 \mathrm{~K})^{17-20}$.

\section{RESULTS AND DISCUSSIONS}

The densities and relative viscosities of all the solutions of nicotinic acid in D-lactose + water systems for four different compositions $(0.05,0.15$, 0.25 and 0.35 ) mol. $\mathrm{kg}^{-1}$ were determined at $303.15 \mathrm{~K}$. The data of relative viscosity has been analysed with Jones Dole equation ${ }^{21}$.

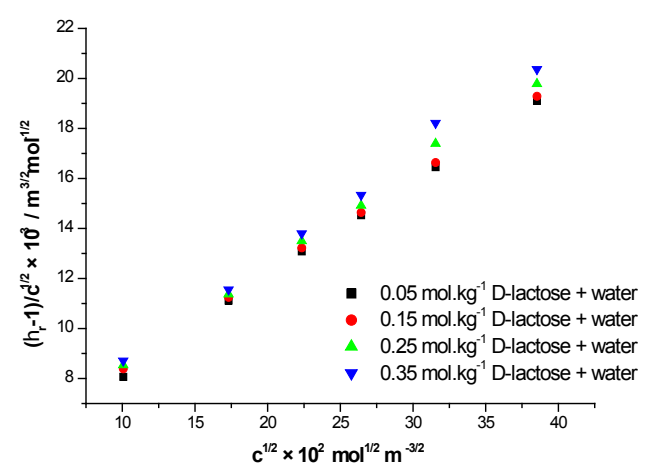

Fig. 1: Plot of $\left(\eta_{\mathrm{r}}-1\right) / \mathbf{c}^{1 / 2} \mathbf{v s} \mathbf{c}^{1 / 2}$ for nicotinic acid in different composition of D-lactose + Water at $303.15 \mathrm{~K}$.

Table1 : Values of A and B coefficient of the Jones - Dole equation , $\overline{V_{1}^{0}}$ (mean volume of the solvent), $V_{2}^{0}$ (partial molar volume), $\Delta \mu_{1}^{0}$ ( free energies of activations per mole of pure solvent) and $\Delta \mu_{2}^{0}$ ( free energies of activations per mole of nicotinic acid) in different composition of D-lactose + Water at 303.15K

\begin{tabular}{llccccc}
\hline $\mathbf{m}^{\mathrm{a}}\left(\mathbf{m o l ~ k g}^{-1}\right)$ & $\mathbf{A}\left(\mathbf{m}^{3 / 2} \mathbf{m o l}^{-1 / 2}\right)$ & $\mathbf{B}\left(\mathbf{m}^{3} \mathrm{~mol}^{-1}\right)$ & $\mathbf{V}_{\mathbf{1}}{ }^{\circ}\left(\mathbf{m}^{3} \mathbf{m o l}^{-1}\right)$ & $\mathbf{V}_{2}{ }^{\circ}\left(\mathbf{m}^{3} \mathrm{~mol}^{-1}\right)$ & $\Delta \boldsymbol{\mu}_{1}{ }^{\circ}\left(\mathbf{k J m o l}^{-1}\right)$ & $\Delta \boldsymbol{\mu}_{2}{ }^{\circ}\left(\mathbf{k J m o l}^{-1}\right)$ \\
\hline 0.05 & $4.349( \pm 0.141)$ & $0.384( \pm 0.005)$ & 18.86 & 81.63 & 26.74 & 61.73 \\
0.15 & $4.340( \pm 0.072)$ & $0.387( \pm 0.002)$ & 20.69 & 81.97 & 26.89 & 60.87 \\
0.25 & $4.230( \pm 0.199)$ & $0.404( \pm 0.007)$ & 23.02 & 82.26 & 27.06 & 61.84 \\
0.35 & $4.181( \pm 0.367)$ & $0.421( \pm 0.013)$ & 26.00 & 82.59 & 27.23 & 62.86 \\
\hline
\end{tabular}




$$
\eta_{r e l}=\frac{\eta}{\eta_{0}}=1+A C^{1 / 2}+B C
$$

Where, $\eta$ is viscosity of solutions and $\eta_{0}$ is viscosity of solvent respectively. $A$ is a constant parameter representing solute-solute interactions where as B is a parameter explains solute solvent interactions. These $A$ and $B$ parameters were obtained by plotting a graph between $\left(\eta_{\mathrm{r}}-1\right) /$ $\mathrm{C}^{1 / 2}$ versus $\mathrm{C}^{1 / 2}$ for nicotinic acid in all the different compositions of D-lactose + water at $303.15 \mathrm{~K}$ and the values are reported in Table 1. Figure 1 represents a sample plot of $\left(\eta_{\mathrm{r}}^{-} 1\right) / C^{1 / 2}$ versus $\mathrm{C}^{1 / 2}$ for nicotinic acid in different compositions of D-Lactose + water.

The A coefficient values reported in table - 1 found to decrease with the increase in the composition of D-lactose in water indicating that solute-solute interactions are weakened with the increase in the composition of D-lactose in water. It

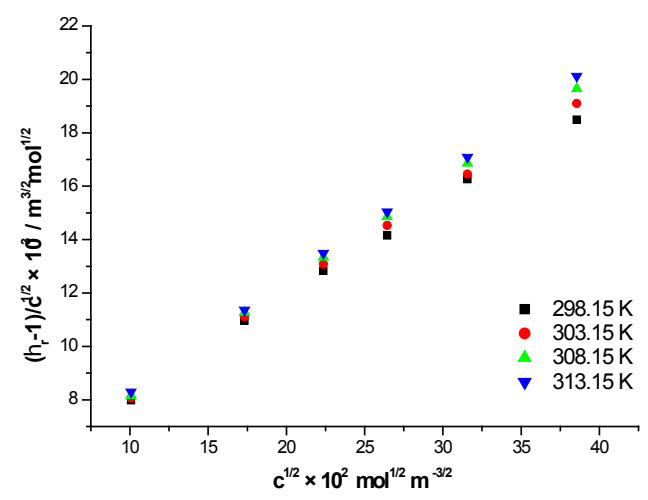

Fig. 2: Plot of $\left(\eta_{\mathrm{r}}-1\right) / \mathbf{c}^{1 / 2} \mathbf{v s} \mathbf{c}^{1 / 2}$ for nicotinic acid in 0.05 mol. $\mathrm{kg}^{-1} \mathrm{D}$-lactose + water at different temperatures. is also found that the B-coefficient values for nicotinic acid are positive, for entire range compositions of D-lactose + water at $303.15 \mathrm{~K}$, indicating the presence of strong solute-solvent interactions, which dominate over the solute-solute interactions. This may be attributed to the strong solvation of these ions by the binary mixtures of D-lactose + water. Further, the $B$-coefficient values increase with the increase of D-lactose content in water at $303.15 \mathrm{~K}$ showing that solute - solvent interactions are further strengthened with the increase of D-lactose content in water indicating that the solvation is further enhanced as the composition of D-lactose increases in water, or it justifies that D-lactose has more affinity for nicotinic acid than that for water.

Feakins theory has also been used to analyse the viscosity data ${ }^{22}$.The transition state theory gives the B parameter by the following relation:

$$
B=\frac{\overline{V_{1}^{0}}-\overline{V_{2}^{0}}}{1000}+\frac{\overline{V_{1}^{0}}}{1000}\left[\frac{\Delta \mu_{2}^{0}-\Delta \mu_{1}^{0}}{R T}\right]
$$

Where, $\overline{V_{1}^{0}}$ is mean volume of the solvent $\overline{V_{2}^{0}}$ is the partial molar volume of the nicotinic acid, is the free energies of activations per mole of pure solvent and is the free energies of activations per mole of the nicotinic acid respectively

$$
\begin{aligned}
& \Delta \mu_{1}^{0}=R T \operatorname{In}\left(\eta_{0} \overline{V_{1}^{0}} / h N\right) \\
& \Delta \mu_{2}^{0}=\Delta \mu_{1}^{0}+R T / \overline{V_{1}^{0}}\left[1000 B-\left(\overline{V_{1}^{0}}-\overline{V_{2}^{0}}\right)\right]
\end{aligned}
$$

Where $\eta_{0}$ is the viscosity of the solvent, $T$ is the absolute temperature, $\mathrm{R}$ is the gas constant,

Table 2: Values of A and B coefficient of the Jones - Dole equation $\overline{V_{1}^{0}}$ (mean volume of the solvent), $V_{2}^{0}$ (partial molar volume), $\Delta \mu_{1}^{0}$ ( free energies of activations per mole of pure solvent) and $\Delta \mu_{2}^{0}$ ( free energies of activations per mole of nicotinic acid) in 0.05 mol.kg-1 D-lactose + water at different temperatures

\begin{tabular}{lcccccc}
\hline $\mathrm{T} / \mathbf{K}$ & $\mathbf{A}\left(\mathbf{m}^{3 / 2} \mathbf{m o l}^{-1 / 2}\right)$ & $\mathbf{B}\left(\mathbf{m}^{3} \mathbf{m o l}^{-1}\right)$ & $\mathbf{V}_{1}{ }^{{ }^{\prime}}\left(\mathbf{m}^{3} \mathbf{m o l}^{-1}\right)$ & $\mathbf{V}_{\mathbf{2}}{ }^{\circ}\left(\mathbf{m}^{3} \mathbf{m o l}^{-1}\right)$ & $\Delta \boldsymbol{\mu}_{1}{ }^{\circ}\left(\mathbf{k J m o l}{ }^{-1}\right)$ & $\Delta \boldsymbol{\mu}_{2}{ }^{\circ}\left(\mathbf{k J m o l}{ }^{-1}\right)$ \\
\hline 298.15 & $4.444( \pm 0.209)$ & $0.369( \pm 0.008)$ & 18.25 & 81.38 & 26.40 & 58.73 \\
303.15 & $4.349( \pm 0.141)$ & $0.384( \pm 0.005)$ & 18.27 & 81.63 & 26.71 & 61.73 \\
308.15 & $4.249( \pm 0.121)$ & $0.401( \pm 0.004)$ & 18.30 & 82.36 & 27.02 & 65.12 \\
313.15 & $4.188( \pm 0.094)$ & $0.412( \pm 0.003)$ & 18.34 & 82.80 & 27.37 & 67.68 \\
\hline
\end{tabular}


$\mathrm{N}$ is the Avogadro number and $\mathrm{h}$ is the Planck's constant.

The values of $\Delta \mu_{1}{ }^{0}$ were calculated with the help of relation (4) and reported in Table 2 .

$$
\overline{V_{1}^{0}}=\left(x_{1} M_{1}+x_{2} M_{2}\right) / \rho_{1}
$$

Wherex ${ }_{1}, M_{1}$ are the mole fractions and molecular weights of the water and $x_{2}, M_{2}$ the same for D-lactose + water respectively where as $\rho_{1}$ is the density of D-lactose + water. The $\overline{V_{2}^{0}}$ values for the nicotinic acid are recorded in Table 1 and calculated with the help of the density data. The $\overline{V_{1}{ }^{0}}$ and $\Delta \mu_{2}{ }^{0}$ values are recorded in Table 1 and were calculated with the help of relations (5) and (6). It is found that the values of $\Delta \mu_{2}^{0}$ and $\overline{V_{1}^{0}}$ are practically constant in all solvent compositions.

It is found that the $\Delta \mu_{2}{ }^{0}$ values are positive and more in magnitude than $\Delta \mu_{1}{ }^{0}$ values which suggests that the transition state formation is less favoured in the presence of the selected nicotinic acid indicating the formation of transition state and is followed by the breaking and distortions of the intermolecular bonds between D-lactose and water. The $\mathrm{dB} / \mathrm{dT}$ is a the important criterion for elucidating the structure making/breaking nature of any solute ${ }^{23}$. The analyses of $\mathrm{dB} / \mathrm{dT}$ parameter was done by studying the effect of temperature.

\section{Effect of temperature}

As the behaviour of nicotinic acid in all different compositions $(0.05,0.15,0.25$ and 0.35$)$

Table 3: Values of $\left(\Delta \mu_{2}{ }^{0}-\Delta \mu_{1}{ }^{0}\right), T \Delta S_{2}{ }^{0}$ and $\Delta \mathbf{H}_{2}{ }^{\circ}$ for nicotinic acid in 0.05 mol. $\mathrm{kg}^{-1} \mathrm{D}$-lactose+ water at different temperatures

\begin{tabular}{crrr}
\hline $\mathbf{T} / \mathbf{K}$ & $\begin{array}{c}\left(\Delta \boldsymbol{\mu}_{2}{ }^{\circ}-\Delta \boldsymbol{\mu}_{1}{ }^{*}\right) \\
\left(\mathbf{k J ~ m o l}{ }^{-1}\right)\end{array}$ & $\begin{array}{r}\mathrm{T} \Delta \mathbf{S}_{2}{ }^{\circ} \\
\left(\mathbf{k J ~ m o l}^{-1}\right)\end{array}$ & $\begin{array}{c}\Delta \mathbf{H}_{2}{ }^{\circ} \\
\left(\mathbf{k J ~ m o l}^{-1}\right)\end{array}$ \\
\hline 298.15 & 32.33 & -180.08 & -121.35 \\
303.15 & 35.02 & -183.10 & -121.37 \\
308.15 & 38.10 & -186.12 & -121.00 \\
313.15 & 40.30 & -189.14 & -121.47 \\
\hline
\end{tabular}

molkg-1 of D-lactose + water was found to be identical and linear at $303.15 \mathrm{~K}$. So, only lower composition i.e 0.05 mol. $\mathrm{kg}^{-1} \mathrm{D}$-lactose + water system has been selected for observing the effect of temperature $(298.15,303.15,308.15$ and 313.15) K. The plot of $\left(\eta_{r^{-}} 1\right) / C^{1 / 2}$ verses $C^{1 / 2}$ has been found to be linear for nicotinic acid at different temperatures. Figure 2 represents the sample plot for nicotinic acid in 0.05 mol. $\mathrm{kg}^{-1} \mathrm{D}$-lactose + water system at different temperatures.

It is found that the values of $A$ parameter that are reported in table 2 , decrease with the rise in temperature in 0.05 mol. $\mathrm{kg}^{-1} \mathrm{D}$-lactose + water for the nicotinic acid. So, it suggests that solute-solute interactions further decreases with the increase of temperature, in other words, the presence of weak solute-solute interactions may be attribute to increase in the solvation of nicotinic acid in D-lactose + water system. From Table 2, the values of B-coefficient are positive for nicotinic acid in 0.05 mol. $\mathrm{kg}^{-1} \mathrm{D}$-lactose + water at different temperatures and it indicates the presence of strong solute-solvent interactions. Further the values of B-coefficient increases with the increase in temperature indicating the enhancement of solute-solvent interaction with rise in temperature. The value of $\mathrm{dB} / \mathrm{dT}$ is found to be negative for nicotinic acid in 0.05 mol. $\mathrm{kg}^{-1}$ D-lactose + water. This indicates the structure making nature of nicotinic acid in D-lactose + water system.

The transition state theory has been applied for interpreting B-coefficients parameter at 298.15, 303.15, 308.15 and 313.15 K. The values of $\Delta \mu_{1}{ }^{0}$ and $\Delta \mu_{2}{ }^{0}$ have been recorded in Table 2 . The quantity $\left(\Delta \mu_{2}{ }^{0}-\Delta \mu_{1}{ }^{0}\right)$,the change in activation energy per mole of solute on replacing one mole of solute by one more mole of solute at infinite dilution large and positive for nicotinic acid in 0.05 mol. $\mathrm{kg}^{-1} \mathrm{D}$-lactose + water at different temperatures and are reported in table 3. Finally, it concludes that the formation of the transition state is followed by breakage of the intermolecular bonds of the solvent.

The entropy of activation $\Delta \mathrm{s}_{2}{ }^{0}$ for nicotinic acid has been determined from the following equations

$$
\frac{d\left(\Delta \mu_{2}^{0}\right)}{d T}=-\Delta S_{2}^{0}
$$


The values of $\Delta \mathrm{s}_{2}{ }^{0}$ have been obtained from the slopes of linear plot of $\Delta \mu_{2}{ }^{0}$ verses $T$ and the enthalpy of activation $\Delta \mathrm{H}_{2}{ }^{0}$ was calculated using relation:

$$
\Delta H_{2}^{0}=\Delta \mu_{2}^{0}+T \Delta S_{2}^{0}
$$

The values of $\mathrm{T} \Delta \mathrm{S}_{2}{ }^{0}$ and $\Delta \mathrm{H}_{2}{ }^{0}$ at different temperatures are reported in Table 3.

It is clear from Table 3 that both parameters enthalpy and entropy of activation are negative for the nicotinic acid indicating that the transition state is more associated with making of bond and increase in order $^{24}$.

\section{CONCLUSION}

The analysed data of relative viscosities of nicotinic acid in different compositions of D-lactose+ water along with the effect of temperature justifies the increase in solute solvent interactions with increase in composition of D-lactose in water as well as with increase in temperature. The negative value of $\mathrm{dB} /$ dT and the transitions state theory supports that structure making nature of nicotinic acid in binary aqueous mixtures of D-lactose.

\section{REFERENCES}

1. Strokes, R.H.; Mills, R. "viscosity of electrolytes and related properties", (Pergman Press, New York), 1965.

2. Nightingale, R.R. in "Chemical Physics of ionic solutions" edited by B.E.Conway and R.G Barrades", (John Wiley, New York), 1965.

3. Kay, R.L.; in Water, a Comprehensive treastise, edited by Franks F (Plenum Press, New York), 1973.

4. Desnoyers, J.E.; Parron G.; J Sol Chem. 1972, 1,199.

5. Parmar, M.L.; Sharma, S. Res. J Chem Environ. 1998, 2, 17.

6. Pandey, J.D.; Akhtar, Y.; Sharma, A.K. Indian J Chem. 1998, A37, 1094.

7. Parmar, M.L.; J Indian Council Chem. 2002, 19, 24.

8. Parmar, M.L.; Dhiman, D.K.; Thakur, R.C. Indian J Chem. 2002, A41, 2032.

9. Parmar, M.L.; Thakur, R.C. Indian J.Chem, 2006, 45(A), 1631.

10. Das, D.; Das, B.; Hazra, D.K. J SolnChem .2002, 31, 425.

11. Parmar, M.L.; Awasthi, R.K.; Guleria, M.K. Indian J Chem. 2004, A43,41.

12. Ali, A.; Shahjahan. J Iranian chem society. 2006, 3, 340-350.
13. Roy, M.N.; Dakua, V.K.; Sinha B. Int $J$ Thermophys. 2007, 28, 1275-1284.

14. Thakur R C.; Sharma, Ravi.; Kumar, Ashish ; Parmar, M.L. J. Mater. Environ. Sci., 2015, 6 (5), 1330.

15. Thakur, R.C.; Sharma, Ravi.; Annu .; Kumar, Ashish. Journal of Chemical and Pharmaceutical Research, 2015, 7(1) ,255.

16. Saini, Balwinder.; Sharma, Ravi.; Thakur, R C. Int.J. ChemTech Res. 2015, 8(12), 395.

17. Coetzee, J. F.; Chang, T. Pure \& Appl. Chem. 1985, 57, 4635

18. Ward, G.K .; Millero F.J. J Solution Chem. 1974, 3, 417.

19. Parmar, M.L.; Khanna A. J Phys Soc Japan. 1986 , 55, 4122.

20. Parmar, M.L.; Khanna, A.; Gupta, V K. Indian J Chem . 1989, 28A, 565.

21. Jones G., Dole M., J Am Chem Soc. 1929, 512950.

22. Feakins, D.; Freemental, J.D.; Lawrence, K.G.; J. Chem. Soc Faraday Trans1. 1974, 70, 795.

23. Sharma, T.S.; Ahuwalia, J.C. Rev ChemSoc.1973, 2, 217.

24. Pal, A.; Kumar, S. Indian J Chem . 2005, 4A, 469. 